\title{
Spinocerebellar degeneration-corneal dystrophy syndrome
}

INSERM

\section{Source}

INSERM. (1999). Orphanet: an online rare disease and orphan drug data base. Spinocerebellar degeneration-corneal dystrophy syndrome. ORPHA:3177

A rare, genetic, neurological disorder characterized by the association of slowly progressive spinocerebellar degeneration and corneal dystrophy, manifesting with bilateral corneal opacities (which lead to severe visual impairment), mild intellectual disability, ataxia, gait disturbances, and tremor. Additional manifestations include facial dysmorphism (i.e. triangular face, ptosis, low-set, posteriorly ang ulated ears, and micrognathia), as well as mild upper motor neuron involvement with hypertonia, lower limb hyperreflexia and extensor plantar responses. There have been no further descriptions in the literature since 1985. 